\title{
A Mathematical Model of Antibody-Dependent Cellular
}

\section{Cytoxicity (ADCC)}

F. Hoffman ${ }^{1}$, D. Gavaghan ${ }^{1}$, J. Osborne ${ }^{1 * 2}$, I.P. Barrett ${ }^{3}$, T. You ${ }^{4 * 5}$,

H. Ghadially ${ }^{6}$, R. Sainson ${ }^{6 *}$, R.W. Wilkinson ${ }^{6}$, H.M. Byrne ${ }^{1,7, \dagger}$,

${ }^{1}$ Department of Computer Science, University of Oxford, Oxford, UK.

${ }^{2}$ School of Mathematics and Statistics, University of Melbourne, AUS.

${ }^{3}$ Quantitative Biology, Astrazeneca Ltd., Cambridge, UK.

${ }^{4}$ Computational Biology, Astrazeneca Ltd., Alderley Edge, UK.

${ }^{5}$ Beyond Consulting Ltd., BioHub, Alderley Park, Cheshire SK12 4TG, UK.

${ }^{6}$ Medimmune Ltd., Cambridge, UK.

${ }^{7}$ Mathematical Institute, University of Oxford, Oxford, UK.

* indicates formerly

${ }^{\dagger}$ Corresponding author. Tel: +44 1865273525.

E-mail address: helen.byrne@maths.ox.ac.uk (H.M. Byrne)

April 28, 2017 


\begin{abstract}
Immunotherapies exploit the immune system to target and kill cancer cells, while sparing healthy tissue. Antibody therapies, an important class of immunotherapies, involve the binding to specific antigens on the surface of the tumour cells of antibodies that activate natural killer (NK) cells to kill the tumour cells. Preclinical assessment of molecules that may cause antibody-dependent cellular cytotoxicity (ADCC) involves co-culturing cancer cells, NK cells and antibody in vitro for several hours and measuring subsequent levels of tumour cell lysis. Here we develop a mathematical model of such an in vitro ADCC assay, formulated as a system of time-dependent ordinary differential equations and in which NK cells kill cancer cells at a rate which depends on the amount of antibody bound to each cancer cell. Numerical simulations generated using experimentally-based parameter estimates reveal that the system evolves on two timescales: a fast timescale on which antibodies bind to receptors on the surface of the tumour cells, and NK cells form complexes with the cancer cells, and a longer time-scale on which the NK cells kill the cancer cells. We construct approximate model solutions on each timescale, and show that they are in good agreement with numerical simulations of the full system. Our results show how the processes involved in ADCC change as the initial concentration of antibody and NK-cancer cell ratio are varied. We use these results to explain what information about the tumour cell kill rate can be extracted from the cytotoxicity assays.
\end{abstract}

Keywords: immunotherapy; antibody-dependent cellular cytotoxicity; ordinary differential equations; asymptotic analysis.

\title{
Highlights
}

- A time-dependent ODE model of antibody-dependent cellular cytotoxicity was developed

- Asymptotic analysis of the model reveals a natural separation of timescales

- Model analysis shows how tumour cell kill rate can be estimated from ADCC experiments 


\section{Introduction}

The immune system plays a key role in cancer progression [2], with evasion of the immune system now recognised as one of the hallmarks of cancer [14]. The design of drugs that manipulate the immune system has the potential to revolutionise treatments for cancer [15]. Antibodybased treatments represent an important class of cancer immunotherapies. Of interest here are antibodies, that induce cancer cell death by binding to specific cell-surface molecules on cancer cells (tumour associated antigens), which interfere with cell signalling and/or flag cells for removal by the immune cells, including natural killer (NK) cells [26]. NK cells establish dynamic contacts with potential target cells and survey their surface for molecules such as antibodies, using receptors with binding specificity for a part of the antibody known as the Fc region. When an NK cell's Fc receptors (FcRs) detect a critical level of antibody, it lyses the cell by the targeted delivery of cytotoxic proteins: this process is termed antibody dependent cellular cytotoxicity (ADCC). It enables individual NK cells sequentially to kill multiple target cells [7, while sparing healthy cells that do not express the relevant antigens.

ADCC is known to contribute to the clinical efficacy of anti-cancer antibodies [17] and it is anticipated that antibodies that enhance ADCC will play a prominent role in the development of future treatments for cancer patients. Experimentalists investigate the capacity of an antibody to induce ADCC by performing in vitro cytotoxicity assays, that measure target cell lysis as antibody concentrations and NK-cancer cell ratios are varied. In these assays, cancer cells, antibodies and NK cells are co-cultured in a micro-well and levels of tumour cell lysis are then measured 2-4 hours later. In a typical assay NK cells and cancer cells are co-cultured at ratios that range from $1: 1$ to $20: 1$, while antibody concentrations vary between $10^{-6} \mu \mathrm{g} / \mathrm{mL}$ and 10 $\mu \mathrm{g} / \mathrm{mL}$ (for more details, see, for example, [24, 28]) .

In Figure 1 we present results from specific cytotoxicity assays in which HER2/neu positive, 


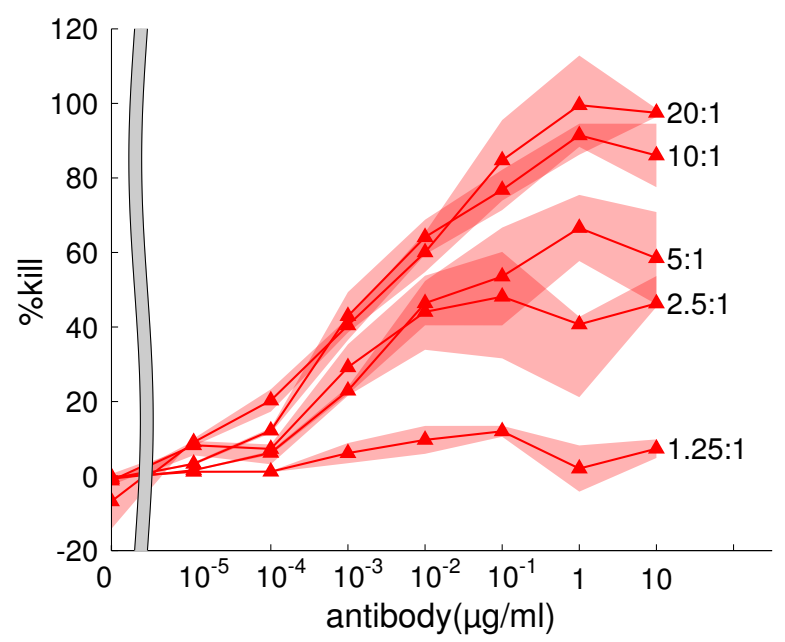

Figure 1: Data from a series of cytotoxicity assays in which HER2/neu positive, SkBr3 breast cancer cells [1] were co-cultured with NK cells in the presence of the antibody Trastuzumab [16. The percentage of cell kill after 4 hours of co-culture was measured for different initial NK-tumour cell ratios $(20: 1,10: 1,5: 1,2: 1,1.25: 1)$ and for a range of initial antibody levels $\left(10^{-6} \mu \mathrm{g} / \mathrm{ml}\right.$ to $\left.10 \mu \mathrm{g} / \mathrm{ml}\right)$. The percentage tumour cell kill associated with an initial antibody level (and an initial NK-tumour cell ratio) was measured as described in the Appendix, all values being measured at the end of the experiment ( $t=4$ hours). Key: mean percentage kill (red triangles); maximum and minimum of triplicate measures for a particular experiment (transparent red fields).

SkBr3 breast cancer cells [11] were co-cultured with NK cells in the presence of the antibody trastuzumab [16]. Briefly, different ratios of effector cells (NK cells) and tumour cells (target 
after four hours of co-culture using a marker of cell lysis (release of an enzyme, GAPDH, into the culture medium). In this way, the percentage of tumour cells killed for a range of different effector/target cell ratios and antibody concentrations can be measured. (Full details of the experimental procedure used to generate the data presented in Figure 1 are provided in Appendix A Figure 1 shows that the percentage kill increases with the initial antibody level and the initial NK-cancer cell ratio. We note also that the cytotoxicity assays provide information about percentage tumour cell kill at a single time-point only ( $t=4$ hours).

Mathematical models represent a natural framework within which to study the processes involved in the cytotoxicity assays and the timescales on which they act. To date, two main approaches have been used to model immune cell killing in cytotoxicity assays: deterministic models, formulated as systems of time-dependent, ordinary differential equations (ODEs) [29, 30, 35, 5], and stochastic models, typically involving Poisson processes [22, 6]. The ODE models can be viewed as mean-field approximations of the stochastic models [13 and are typically more analytically and computationally tractable than their stochastic counterparts. Therefore, in this paper, we use an ODE approach to develop a dynamic, mathematical model of in vitro ADCC. Where existing models of cancer cell killing by immune cells assume a constant kill rate and neglect the role of antibodies in modulating the cell kill rate [29, 30, 35, 5], our model assumes that the rate at which NK cells kill cancer cells depends upon the number of antibodies bound to the target/cancer cells. For completeness, we note that other authors have modelled the relationship between binding parameters and cytotoxicity in ADCC using non-ODE based approaches (see [9, 12] for further details).

We use the experimental literature to estimate parameter values and then nondimensionalise the model equations, before investigating them with a combination of numerical simulations and analytical techniques. Throughout this study, our main aim is to establish what information can be obtained about the way in which ADCC modulates the rate at which NK cells kill tumour 
cells. Our analysis reveals how data from the different cytotoxicity assays may be aggregated to provide valuable information about the rate at which NK cell killing of cancer cells is enhanced by ADCC.

The remainder of the paper is structured as follows. In Section 2 we introduce our mathematical model, nondimensionalise the governing equations and then introduce a change of variables in order to simplify the subsequent analysis. We also use the experimental literature to estimate model parameters and identify a range of parameter scalings relevant to antibody-based cancer therapy. In Section 3 we perform numerical simulations to illustrate how the system dynamics change as the experimental conditions vary. In Section 4 we exploit the parameter scalings identified in Section 2 by using perturbation methods to construct approximate model solutions. The paper concludes in Section 5 with a discussion of our results and suggestions for future work.

\section{A Mathematical Model of ADCC}

In this section we develop a new mathematical model of an ADCC cytotoxicity assay in which cancer cells, antibodies and NK cells are incubated in a micro-well for four hours and then the number of dead target cells is measured (see Figure 1). The model is formulated as a system of six time-dependent ODEs and accounts for processes that occur on the subcellular and cellular scales. At the subcellular scale, the dependent variables represent the concentration of antibodies, $A(t)$ (moles per litre), the concentration of unbound target receptors, $T_{u}(t)$ (moles per litre), and the concentration of antibody-target receptor complexes, $T_{b}(t)$ (moles per litre).

At the cellular scale, the dependent variables represent the concentration of NK cells, $N(t)$, the concentration of cancer cells, $T(t)$, and the concentration of NK-cancer cell complexes, $C(t)$, all variables having units of cells per cubic metre. Our model accounts for the binding of 


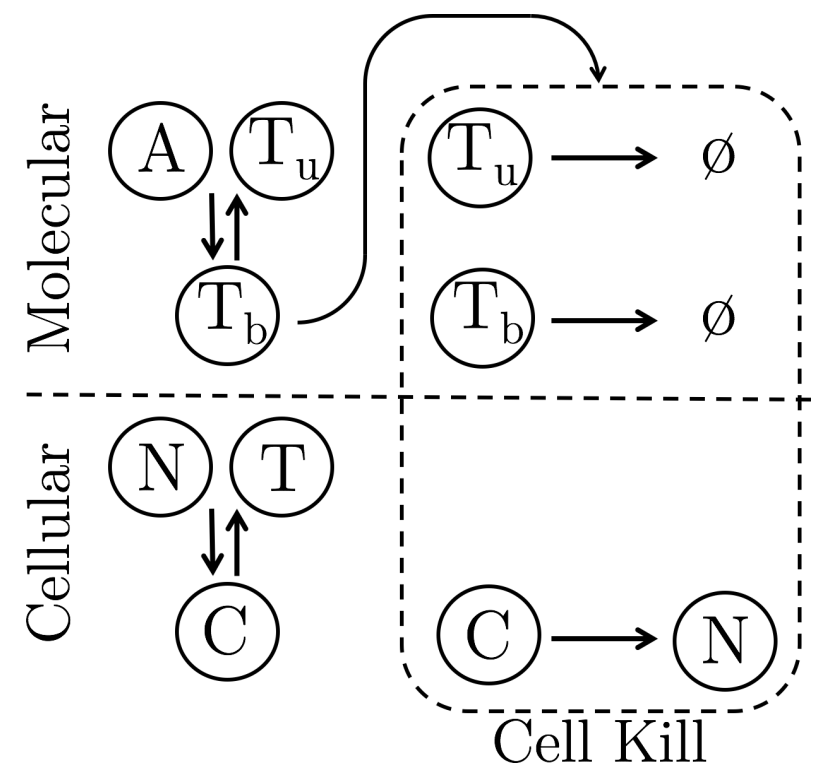

Figure 2: Schematic of the model illustrating the cellular and molecular components.

antibodies to target receptors on cancer cells, NK cell binding to cancer cells, and NK cell killing of cancer cells in response to antibody-bound targets. A schematic of the model is presented in Figure 2 .

As the time-scale of experiments (4 hours) is much shorter than typical cell cycle times of NK cells [18] and cancer cells [23], we neglect processes such as cell division and natural cell death, which operate on longer time-scales. We assume further that the cells and antibody are well-mixed so that spatial effects may be neglected. While direct evidence to justify a wellmixed model is currently lacking, our assumption is based on: (a) the volumes used are small and so diffusion should not be limiting during the timescales of the experiments, (b) we are not adding any further components (e.g. lipid particles) that may plausibly sequester the species considered and (c) no obvious precipitation is observed visually. Future studies are needed to verify these assumptions.

By applying the principle of mass balance to each dependent variable and appealing to the 
Law of mass action, we arrive at the following system of ODEs:

$$
\begin{aligned}
& \frac{d A}{d t}=-\underbrace{k_{\mathrm{on}} T_{u} A}_{\text {Ab. target assoc. }}+\underbrace{k_{\mathrm{off}} T_{b}}_{\text {Ab. target dissoc. }}, \\
& \frac{d T_{u}}{d t}=-k_{\mathrm{on}} T_{u} A+k_{\mathrm{off}} T_{b}-\underbrace{f\left(\frac{T_{b}}{T+C}\right) \frac{T_{u}}{T+C} C}_{\text {target removal }}, \\
& \frac{d T_{b}}{d t}=k_{\mathrm{on}} T_{u} A-k_{\mathrm{off}} T_{b}-\underbrace{f\left(\frac{T_{b}}{T+C}\right) \frac{T_{b}}{T+C} C}_{\text {Ab./target complex removal }}, \\
& \frac{d N}{d t}=-\underbrace{\beta_{\text {on } N T}}_{\text {NK/T assoc. }}+\underbrace{\beta_{\text {off }} C}_{\text {NK/T dissoc. }}+\underbrace{f\left(\frac{T_{b}}{T+C}\right) C}_{\text {NK dissoc. after kill }}, \\
& \frac{d T}{d t}=-\beta_{\mathrm{on}} N T+\beta_{\mathrm{off}} C, \\
& \frac{d C}{d t}=\beta_{\mathrm{on}} N T-\beta_{\mathrm{off}} C-\underbrace{f\left(\frac{T_{b}}{T+C}\right) C}_{\text {NK/T killing }} .
\end{aligned}
$$

Equations (1)-(3) define the subcellular dynamics. Antibodies, $A$, are assumed to bind reversibly to unbound tumour receptors, $T_{u}$, at rate $k_{\text {on }}$, forming bound tumour receptors, $T_{b}$, from which they dissociate at rate $k_{\text {off }}$. We assume antibody is removed from the system upon tumour cell death, via local release of proteolytic enzymes from the dying cell. We have no direct evidence underpinning this assumption, but we feel it is reasonable as established protein preparation protocols routinely employ proteolytic enzyme inhibitors to avoid degradation of protein from lysed cells. Equations (4)-(6) define the cellular dynamics. NK cells, $N$, are assumed to bind reversibly to cancer cells, $T$, at rate $\beta_{\text {on }}$, forming cellular conjugates, $C$, from which they dissociate at rate $\beta_{\text {off }}$. While Deguine et al. [8] report that antibody recognition by NK cells increases contact stability with cancer cells, for simplicity, we assume that the rates of NK-cancer cell association and dissociation are independent of bound antibodies $\left(T_{b}\right)$. We postpone to future work a comparison of this case with one in which $\beta_{\text {on }}$ and $\beta_{\text {off }}$ depend on $T_{b} /(T+C)$, the number of antibody-target receptor complexes per cancer cell.

The subcellular and cellular dynamics are coupled by NK cell-mediated killing of the tumour 
cells. We suppose that NK cells bound to cancer cells kill the cancer cells at a rate, $f\left(\frac{T_{b}}{T+C}\right)$, which is a monotonically increasing, saturating function of $\frac{T_{b}}{T+C}$, the number of antibody-target receptor complexes per cancer cell. We assume further that $f(0)=0$ so that no killing occurs in the absence of bound antibody. As an illustrative example, we will use the following functional form for $f$,

$$
f(R)=\frac{f_{K}^{*} R}{R+R_{K}}
$$

where $f_{K}^{*}$ represents the maximum rate of killing and $R_{K}$ is the value of $R=\frac{T_{b}}{T+C}$ at which the rate of killing is half-maximal. However, since much of the analysis presented in the remainder of the paper holds for more general choices for $f$ (with $f(0)=0$ ), where appropriate we will use the general form.

After a tumour cell has been killed, NK cells can detach from their target and then move on to another target cell (see: Bhat and Watzl [3]). We model this effect by supposing that when a tumour cell has been killed, the NK cell dissociates, giving rise to a source term in Equation (4). Cancer cell killing is also accompanied by a reduction in unbound and antibody bound receptors. For simplicity, we assume that all cancer cells have the same number of receptors, which we denote by $\rho_{T}$, and, hence, that cancer cell death will reduce receptor numbers at a rate proportional to the cell death rate. Since the numbers of unbound and bound receptors per cancer cell are $\frac{T_{u}}{T+C}$ and $\frac{T_{b}}{T+C}$ respectively, we assume that unbound and bound receptors are removed from the system at rates $f\left(\frac{T_{b}}{T+C}\right) \frac{T_{u}}{T+C} C$ and $f\left(\frac{T_{b}}{T+C}\right) \frac{T_{b}}{T+C} C$, respectively (see Equations (2) and (3)).

We close Equations (1)-(6) by imposing suitable initial conditions. At the start of the experiment, antibodies, NK cells and cancer cells are added separately to a micro-well. Therefore we assume that at $t=0$ no antibodies are bound to targets $\left(T_{b}(0)=0\right)$ and no NK cells are bound to cancer cells $(C(0)=0)$. We denote by $A_{0}, N_{0}$ and $T_{0}$ the initial concentrations of 
antibodies, cancer cells and NK cells so that $A(0)=A_{0}, N(0)=N_{0}$ and $T(0)=T_{0}$ and assume that initially all target receptors are unbound, so that $T_{u}(0)=\rho_{T} T_{0}$. To summarise, the initial conditions that close Equations (1)-(6) are

$$
A(0)=A_{0}, \quad T_{u}(0)=\rho_{T} T_{0}, \quad T_{b}(0)=0, \quad N(0)=N_{0}, \quad T(0)=T_{0} \text { and } C(0)=0 .
$$

\subsection{Model Reduction, Nondimensionalisation and Variable Transformation}

Inspection of Equations (1)-(6) reveals two conserved quantities which we may exploit to reduce our model from six equations to four. Firstly, adding Equations (4) and (6) and integrating subject to Equation (8) supplies

$$
N+C=N_{0} .
$$

Equation (9) states that the total concentration of NK cells (bound and unbound) remains constant during the experiment. Secondly, combining Equations (2), (3), (5) and (6) and exploiting Equation (8) gives

$$
T_{u}+T_{b}=\rho_{T}(T+C),
$$

reflecting the fact that, at any given time, the total concentration of target receptors (free and bound) is proportional to the total concentration of cancer cells (free and in complex), with constant of proportionality $\rho_{T}$. We exploit these conservation laws to eliminate $N$ and $T_{u}$ from Equations (1)-(8), which reduce to give,

$$
\begin{aligned}
\frac{d A}{d t} & =-k_{\mathrm{on}}\left(\rho_{T}(T+C)-T_{b}\right) A+k_{\mathrm{off}} T_{b} \\
\frac{d T_{b}}{d t} & =k_{\mathrm{on}}\left(\rho_{T}(T+C)-T_{b}\right) A-k_{\mathrm{off}} T_{b}-f\left(\frac{T_{b}}{T+C}\right) \frac{T_{b}}{(T+C)} C \\
\frac{d T}{d t} & =-\beta_{\mathrm{on}}\left(N_{0}-C\right) T+\beta_{\mathrm{off}} C \\
\frac{d C}{d t} & =\beta_{\mathrm{on}}\left(N_{0}-C\right) T-\beta_{\mathrm{off}} C-f\left(\frac{T_{b}}{T+C}\right) C
\end{aligned}
$$

with $A(0)=A_{0}, T_{b}(0)=0, T(0)=T_{0}$ and $C(0)=0$. 


$$
R(t)=\frac{T_{b}}{T+C} \text { and } S(t)=T+C .
$$

Here $R(t)$ is the number of antibody-receptor complexes per cancer cell and $S(t)$ is the total concentration of cancer cells (free and bound to NK cells). Under this change of variables, Equations (11)-(15) become

$$
\begin{aligned}
\frac{d A}{d t} & =-k_{\mathrm{on}}\left(\rho_{T} S-R S\right) A+k_{\mathrm{off}} R S, \\
\frac{d R}{d t} & =k_{\mathrm{on}}\left(\rho_{T}-R\right) A-k_{\mathrm{off}} R, \\
\frac{d S}{d t} & =-f(R) C, \\
\frac{d C}{d t} & =\beta_{\mathrm{on}}\left(N_{0}-C\right)(S-C)-\beta_{\mathrm{off}} C-f(R) C, \\
\text { with } A(0) & =A_{0}, R(0)=0, S(0)=T_{0} \text { and } C(0)=0 .
\end{aligned}
$$

In what follows it will be convenient to recast Equations (17)-21) in dimensionless form using the following rescaling

$$
A=A_{0} A^{*}, R=\rho_{T} R^{*}, S=T_{0} S^{*}, C=T_{0} C^{*}, t=t_{0} t^{*},
$$

where $t_{0}$ is the timescale of the cytotoxicity assay. Under this transformation, Equations (17)(21) become (on dropping the asterisks),

$$
\begin{aligned}
\frac{d A}{d t} & =-\alpha_{1}(1-R) A S+\alpha_{2} \gamma R S, \\
\frac{d R}{d t} & =\frac{\alpha_{1}}{\gamma}(1-R) A-\alpha_{2} R, \\
\frac{d S}{d t} & =-\hat{f}(R) C, \\
\frac{d C}{d t} & =\nu_{1}(\mu-C)(S-C)-\nu_{2} C-\hat{f}(R) C .
\end{aligned}
$$


In Equations (23)-26 we have introduced the following dimensionless parameter groupings and functions

$$
\begin{gathered}
\alpha_{1}=k_{\mathrm{on}} t_{0} \rho_{T} T_{0}, \alpha_{2}=k_{\text {off }} t_{0}, \gamma=\frac{\rho_{T} T_{0}}{A_{0}}, \nu_{1}=\beta_{\text {on }} t_{0} T_{0}, \nu_{2}=\beta_{\text {off }} t_{0}, \mu=\frac{N_{0}}{T_{0}} \\
\text { and } \hat{f}(R)=t_{0} f\left(\rho_{T} R\right) .
\end{gathered}
$$

\section{$2.2 \quad$ Parameter Estimation}

Dimensional and dimensionless estimates of the model parameters are presented in Tables 1 and 2 respectively. The latter are obtained from the former using Equation (27). Details of how these estimates were obtained are presented in Appendix B.

\begin{tabular}{|c|l|l|l|}
\hline Parameter & Definition & Estimated Value $($ units $)$ & Source \\
\hline$\rho_{T}$ & Number of receptors per cell & $10^{3}$ to $10^{7}\left(\right.$ molecule cell $\left.{ }^{-1}\right)$ & {$[34,[19]$} \\
$k_{\text {on }}$ & Antibody binding rate & $10^{3}$ to $10^{7}\left(\mathrm{M}^{-1} \mathrm{~s}^{-1}\right)$ & {$[4,[10]$} \\
$k_{\text {off }}$ & Antibody dissociation rate & $10^{-4}\left(\mathrm{~s}^{-1}\right)$ & {$[4,[10]$} \\
\hline$\beta_{\text {on }}$ & Cell binding rate & $2 \times 10^{13}\left(\mathrm{M}^{-1} \mathrm{~s}^{-1}\right)$ & {$[1]$} \\
$\beta_{\text {off }}$ & Cell dissociation rate & $10^{-3}\left(\mathrm{~s}^{-1}\right)$ & {$[1]$} \\
$f\left(\frac{T_{b}}{T+C}\right)$ & Cell kill function & 0 to $1.6 \times 10^{-3}\left(\mathrm{~s}^{-1}\right)$ & {$[32]$} \\
\hline$A_{0}$ & Initial antibody concentration & $6.67 \times 10^{-12}$ to $10^{-5}(\mathrm{M})$ & \\
$N_{0}$ & Initial NK cell concentration & $4.15 \times 10^{-16}$ to $8.30 \times 10^{-15}(\mathrm{M})$ & {$[24,28]$} \\
\hline$T_{0}$ & Initial tumour cell concentration & $4.15 \times 10^{-16}(\mathrm{M})$ & {$[24,28]$} \\
\hline$t_{0}$ & Time-scale of experiment & $1.44 \times 10^{4}(\mathrm{~s})$ & \\
\hline
\end{tabular}

Table 1: Summary of the parameters associated with Equations (11)-(15), together with estimates of their dimensional values. 


\begin{tabular}{|l|c|l|}
\hline Parameter & Definition & Estimated Value \\
\hline$\alpha_{1}$ & $k_{\text {on }} t_{0} \rho_{T} T_{0}$ & $5.8 \times 10^{-6}$ to $5.8 \times 10^{2}$ \\
$\alpha_{2}$ & $k_{\text {off }} t_{0}$ & 1.44 \\
$\gamma$ & $\frac{\rho_{T} T_{0}}{A_{0}}$ & $6.22 \times 10^{-9}$ to $6.22 \times 10^{2}$ \\
$\nu_{1}$ & $\beta_{\text {on }} t_{0} T_{0}$ & $1.2 \times 10^{2}$ \\
$\nu_{2}$ & $\beta_{\text {off }} t_{0}$ & 14.4 \\
$\mu$ & $\frac{N_{0}}{T_{0}}$ & 1 to 20 \\
$\hat{f}(R)$ & $t_{0} f\left(\frac{\rho_{T} T_{b}}{C+S}\right)$ & 0 to 22.4 \\
\hline
\end{tabular}

Table 2: Summary of the dimensionless parameters associated with Equations 23)-26, together with estimates of their values (see also Equation (27)).

${ }_{151}$ We note from Table 2 that two parameters are typically much smaller than the others: $\alpha_{1}=O\left(10^{-6}\right)$ 152 to $O\left(10^{2}\right)$ and $\gamma=O\left(10^{-9}\right)$ to $O\left(10^{2}\right)$. For ADCC, $\alpha_{1}$ and $\gamma$ are fundamental quantities: $\alpha_{1}$ is the product of the antibody association rate and the total number of target receptors initially present in the system while $\gamma$ is the ratio of the number of target receptors to the number of antibodies initially present in the assay. In Table 3 we state order of magnitude estimates of $\alpha_{1}$ and $\gamma$ for three values of $\rho_{T}$ and $k_{\text {on }}$ that span the range found in the literature $[34,19,4$, 10, In each case, the range of values of $\gamma$ reflects the range of values of $A_{0}$ used in cytotoxicity assays. 


\begin{tabular}{|c|c|c|c|}
\hline & $\rho_{T}=10^{3}$ & $\rho_{T}=10^{5}$ & $\rho_{T}=10^{7}$ \\
\hline$k_{\mathrm{on}}=10^{3}$ & $\begin{array}{l}\alpha_{1}=O\left(10^{-6}\right) \\
\gamma=O\left(10^{-9}\right) \text { to } O\left(10^{-2}\right)\end{array}$ & $\begin{array}{l}\alpha_{1}=O\left(10^{-4}\right), \\
\gamma=O\left(10^{-7}\right) \text { to } O(1)\end{array}$ & $\begin{array}{l}\alpha_{1}=O\left(10^{-2}\right) \\
\gamma=O\left(10^{-5}\right) \text { to } O\left(10^{2}\right)\end{array}$ \\
\hline$k_{\mathrm{on}}=10^{5}$ & $\begin{array}{l}\alpha_{1}=O\left(10^{-4}\right) \\
\gamma=O\left(10^{-9}\right) \text { to } O\left(10^{-2}\right)\end{array}$ & $\begin{array}{l}\alpha_{1}=O\left(10^{-2}\right), \\
\gamma=O\left(10^{-7}\right) \text { to } O(1)\end{array}$ & $\begin{array}{l}\alpha_{1}=O(1) \\
\gamma=O\left(10^{-5}\right) \text { to } O\left(10^{2}\right)\end{array}$ \\
\hline$k_{\mathrm{on}}=10^{7}$ & $\begin{array}{l}\alpha_{1}=O\left(10^{-2}\right) \\
\gamma=O\left(10^{-9}\right) \text { to } O\left(10^{-2}\right)\end{array}$ & $\begin{array}{l}\alpha_{1}=O(1) \\
\gamma=O\left(10^{-7}\right) \text { to } O(1)\end{array}$ & $\begin{array}{l}\alpha_{1}=O\left(10^{2}\right) \\
\gamma=O\left(10^{-5}\right) \text { to } O\left(10^{2}\right)\end{array}$ \\
\hline
\end{tabular}

Table 3: Table showing how the dimensionless parameters $\alpha_{1}=k_{\mathrm{on}} t_{0} \rho_{T} T_{0}$ and $\gamma=\frac{\rho_{T} T_{0}}{A_{0}}$ (see Equation (27) and Table 22 vary with $\rho_{T}$ and $k_{\text {on }}$. The range of values of $\gamma$ mirrors the range in $A_{0}\left(A_{0} \in\left[6.67 \times 10^{-12}, 6.67 \times 10^{-5}\right] \mathrm{M}\right.$; see Table 1$)$.

Of the antibody:cancer cell combinations represented in Table 3 , the first row corresponds to the anti-CD20 antibody rituximab and CD20 expression in B cell cancers [34, 10]. For rituximab, $k_{\text {on }}=O\left(10^{3}\right) \mathrm{M}^{-1} \mathrm{~s}^{-1}$, while target receptor levels in B cell cancers range from $O\left(10^{3}\right)$ to $O\left(10^{7}\right)$ receptors per cell. The parameter values in row 2 (columns 2 and 3 ) apply to the anti-HER2/neu antibody trastuzumab which is used to target HER2/neu expression in breast cancer [19, 4]. Trastuzumab has $k_{\mathrm{on}}=O\left(10^{5}\right) \mathrm{M}^{-1} \mathrm{~s}^{-1}$ and is only prescribed for breast cancers with high HER2/neu expression $\left(\rho_{T}>10^{6}\right.$ receptors per cell). Table 3 reveals that the dimensionless parameter $\alpha_{1}$ may range in value from small to $O(1)$ or larger, and that $\gamma$ may also range from very small to large. We note, however, that for most of the parameter ranges recorded in Table 2 , $\alpha_{1} \ll 1$ and $\gamma \leq O(1)$. Therefore, in what follows we fix $\alpha_{1}=\epsilon \ll 1$ and investigate separately the cases $\gamma=O(1), O(\epsilon)$ and $O\left(\epsilon^{2}\right)$.

Based on Table 2 we note that the (dimensionless) rate at which NK cells bind to cancer cells is large $\left(\nu_{1}=O\left(\epsilon^{-1}\right)\right)$. We will exploit this scaling in the asymptotic analysis presented in Section 4. First, to illustrate the relevance of the scalings to cytotoxicity assays, we fix $\epsilon=10^{-3}$, $\rho_{T}=10^{5}$ and indicate on Figure 3 which cytotoxicity experiments correspond to each of the 
scalings for the dimensionless parameter $\gamma\left(\gamma=O(1), O(\epsilon)\right.$, and $\left.O\left(\epsilon^{2}\right)\right)$.

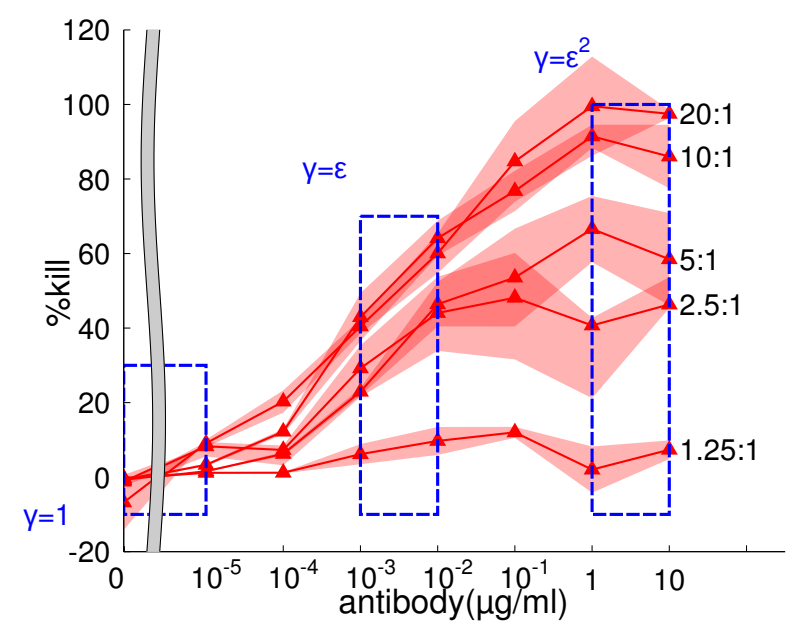

Figure 3: Cytotoxicity data from Figure 1, with the blue rectangles indicating which data corresponds to the three asymptotic cases of interest $\left(\gamma=O(1), O(\epsilon)\right.$ and $\left.O\left(\epsilon^{2}\right)\right)$.

\section{$3 \quad$ Model Dynamics}

Before analysing our model, we present numerical results obtained by integrating Equations (23)-(26), using MATLAB's inbuilt routine ODE15s [20]. ODE15s uses a variable order method based on numerical differentiation formulae and is designed to solve stiff ODEs [27]. For illustrative purposes, we use the dimensionless form of the kill rate function given by Equation (7)

$$
\hat{f}(R)=\frac{f_{K} R}{R+R_{K}}
$$

where $f_{K}$ represents the maximum rate of killing (dimensionless) and $R_{K}$ is the value of $R$ at which the rate of killing is half-maximal. In what follows, we fix $\hat{f}_{K}=1$ and $R_{K}=0.5$. We suppose that $\alpha_{1}=\epsilon=10^{-3}$ and that the initial ratio of NK cells to cancer cells is 20:1. With the exception of $\gamma$, all other parameters are assigned values as per Table 2. The numerical results presented in Figure 4 show how the system dynamics change as $\gamma$ varies. We plot the 
181 time evolution of $R(t), S(t)$ and $C(t)$ (see Equations 23 -26). The dynamics of $A(t)$ are 182 omitted since, for this choice of parameters, $A(t) \approx 1$ for the timescale of interest $(0 \leq t \leq 1)$. 

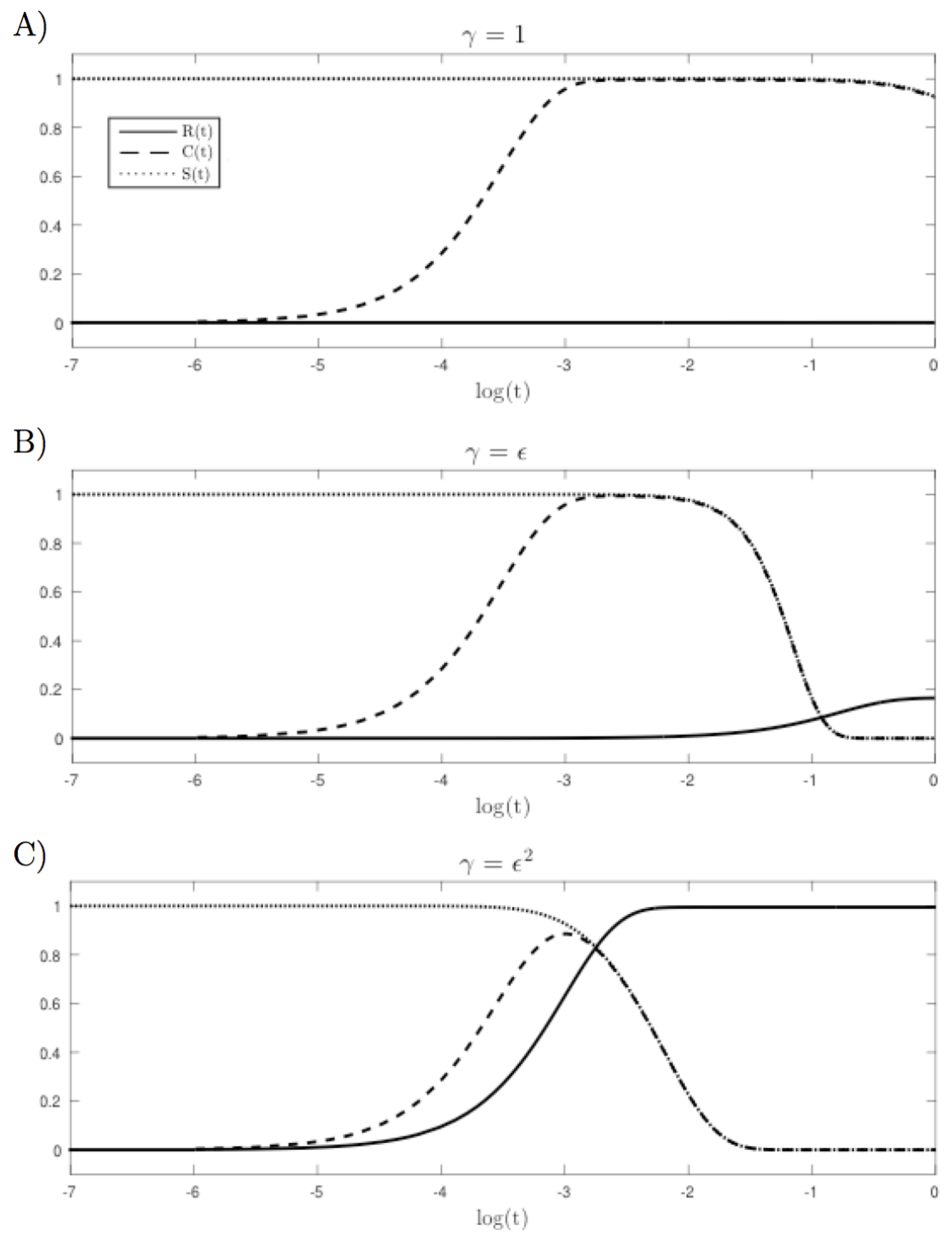

Figure 4: Series of numerical results, obtained by solving the dimensionless Equations 23)26 with $\hat{f}(R)=\frac{f_{K} R}{R+R_{K}}$. The simulation results show how the time evolution of the number of antibody-receptor complexes per cancer cell, $R(t)=\frac{T_{b}}{T+C}$ (solid line), the total concentration of cancer cells, $S(t)=T+C$ (dotted line), and the concentration of cancer cells bound to NK cells, $C(t)$ (dashed line), change as we vary $\gamma$, the ratio of the initial number of target receptors to the initial number of antibodies. Panel (A) corresponds to low antibody levels $(\gamma=1)$, Panel (B) is for intermediate levels of antibody $(\gamma=\epsilon)$ and Panel $(\mathrm{C})$ is for high levels of antibody $\left(\gamma=\epsilon^{2}\right)$. Parameter values: as per Table 2, with $\hat{f}_{K}=1, R_{K}=0.5, \alpha_{1}=\epsilon=10^{-3}, \mu=1$. 
For all values of $\gamma$, at early times (when $0<t<10^{-3}$ ), the total number of cancer cells, $S(t)=T(t)+C(t)$, remains constant $(S(t) \approx 1)$, and cell killing is negligible. During this time, cancer cells form complexes with NK cells $(C(t)$ increases from zero to a maximum value which decreases in magnitude as $\gamma$ decreases). On a longer time-scale, $R(t)$, the number of receptors per cancer cell bound by antibodies, increases at a rate which increases as $\gamma$ decreases (i.e., as antibody levels increase). When $\gamma=\epsilon^{2}$, antibody is plentiful and rapidly binds to all available receptor so that $R(t) \approx 1$ for $t \approx 10^{-2}$. In this case, receptor saturation is sustained for the remainder of the simulation, because the ratio of antibody to target receptors is large. The increase in $R(t)$ stimulates the killing of cancer cells by bound NK cells, so $S(t)$ and $C(t)$ decline over a similar time-scale. This effect is more pronounced for smaller values of $\gamma$. Indeed, for smaller values of $\gamma$ the dynamics associated with $R(t), S(t)$ and $C(t)$ merge (see Figure $4(\mathrm{C}))$. By contrast, when $\gamma=O(1)$, the simulations needs to be continued for longer times in order to see a signficant reduction in the number of cancer cells (see Figure 4(A)).

We emphasise that the main aim of Figure 4 is to show how the dynamic behaviour of the model changes as the dimensionless parameter $\gamma$ varies. Since the experimental data were collected at a single time-point, it is not possible to compare these numerical results to the experimental data. Rather, the numerical results highlight the additional insight that is afforded by developing a dynamic model of ADCC. We note also that the duration of the numerical simulations matches that of the experiments (4 hours in dimensional terms). It is not appropriate to extend the simulations beyond this timescale because processes which have been neglected, such as cell proliferation and natural cell death, become important.

To summarise, the numerical simulations presented in Figure 4 show that the system dynamics act on two different timescales. On the short timescale, tumour cell killing is negligible, NKcancer conjugation approaches equilibrium and, if antibody levels are high enough, the binding of antibodies to target receptors increases towards an equilibrium. On a longer time-scale the 
number of antibodies bound to target receptors becomes large enough to induce NK cells to kill

cancer cells.

\section{Perturbation Analysis}

In this section we focus on the parameter scalings introduced in Section 2.2 and use perturbation methods to construct approximate solutions on the short and long timescales. We recall from Tables 2 and 3 , that $\nu_{1}$, the rate at which NK cells bind to cancer cells, is large while $\alpha_{1}$, the product of the antibody association rate and the total number of target receptors initially present in the system, is typically small. Accordingly, fixing $\alpha_{1}=\epsilon \ll 1$ and $\nu_{1}=\frac{\hat{\nu}_{1}}{\epsilon}$, Equations (23)- 26) supply

$$
\begin{aligned}
\frac{d A}{d t} & =-\left[\epsilon(1-R) A-\alpha_{2} \gamma R\right] S, \\
\frac{d R}{d t} & =\frac{1}{\gamma}\left[\epsilon(1-R) A-\alpha_{2} \gamma R\right], \\
\frac{d S}{d t} & =-\hat{f}(R) C, \\
\frac{d C}{d t} & =\frac{\hat{\nu}_{1}}{\epsilon}(\mu-C)(S-C)-\nu_{2} C-\hat{f}(R) C,
\end{aligned}
$$

with $A(0)=1, R(0)=0, S(0)=1$ and $C(0)=0$.

Equations (30)-(34) define a singular perturbation problem which we analyse on two timescales. We construct an inner solution on a short timescale for which $t=O(\epsilon)$ and match this to an outer solution which evolves on a longer timescale, for which $t=O(1)$. From Figure 4 it is clear that details of the solutions change as $\gamma$ varies. For example, when $0<\gamma \ll 1$ the two timescales merge. While details of the solutions change as $\gamma$ varies, the approach is similar and, therefore, we focus our analysis on the case $\gamma=O(1)$. 
Then Equations (30)-(34) transform to give

$$
\begin{aligned}
\frac{d a}{d \tau} & =-\epsilon\left[\epsilon(1-r) a-\alpha_{2} \gamma r\right] s, \\
\frac{d r}{d \tau} & =\frac{\epsilon}{\gamma}\left[\epsilon(1-r) a-\alpha_{2} \gamma r\right], \\
\frac{d s}{d \tau} & =-\epsilon \hat{f}(r) c, \\
\frac{d c}{d \tau} & =\hat{\nu}_{1}(\mu-c)(s-c)-\epsilon \nu_{2} c-\epsilon \hat{f}(r) c, \\
\text { with } a(0) & =1, r(0)=0, s(0)=1 \text { and } c(0)=0 .
\end{aligned}
$$

We seek trial solutions of the form

$$
a(\tau)=a_{0}(\tau)+\epsilon a_{1}(\tau)+\epsilon^{2} a_{2}(\tau)+O\left(\epsilon^{3}\right),
$$

with similar expansions for $r(\tau), s(\tau)$ and $c(\tau)$. Substituting these trial solutions in Equations (35)-(39) and equating to zero coefficients of $O(1), O(\epsilon)$ and $O\left(\epsilon^{2}\right)$ yields the following approximate solutions for $a, r, s$ and $c$ :

$$
\begin{aligned}
& a(\tau)=1-\epsilon^{2} \tau+O\left(\epsilon^{3}\right), \\
& r(\tau)=\frac{\epsilon^{2} \tau}{\gamma}+O\left(\epsilon^{3}\right), \\
& s(\tau)=1+O\left(\epsilon^{3}\right), \\
& c(\tau)=\mu \frac{\left(1-e^{-\hat{\nu}_{1}(\mu-1) \tau}\right)}{\left(\mu-e^{-\hat{\nu}_{1}(\mu-1) \tau}\right)}+O(\epsilon) .
\end{aligned}
$$


with similar expressions for $R(t), S(t)$ and $C(t)$. Substituting these trial solutions in Equations (30)-(33) and equating to zero coefficients of $O(1)$ and $O(\epsilon)$, it is straightforward to obtain the following expressions for the dependent variables

$$
\begin{aligned}
& A(t)=1-\frac{\epsilon}{\alpha_{2}}\left(1-e^{-\alpha_{2} t}\right)+O\left(\epsilon^{2}\right), \\
& R(t)=\frac{\epsilon}{\alpha_{2} \gamma}\left(1-e^{-\alpha_{2} t}\right)+O\left(\epsilon^{2}\right), \\
& S(t)=1-\frac{\epsilon \hat{f}^{\prime}(0)}{\alpha_{2} \gamma}\left(t-\frac{1}{\alpha_{2}}\left(1-e^{-\alpha_{2} t}\right)\right)+O\left(\epsilon^{2}\right), \\
& C(t)=1-\frac{\epsilon \hat{f}^{\prime}(0)}{\alpha_{2} \gamma}\left(t-\frac{1}{\alpha_{2}}\left(1-e^{-\alpha_{2} t}\right)\right)-\frac{\epsilon \nu_{2}}{\nu_{1}(\mu-1)}+O\left(\epsilon^{2}\right) .
\end{aligned}
$$

As expected from the numerical results presented in Figure 3(a), when $\gamma=O(1)$ and antibody levels are low, tumour cell killing is negligible during the timecourse of the experiments $(t=O(1))$. Equations 477$)-(50)$ reveal how the system dynamics depend on the model parameters and, in particular, on $\hat{f}^{\prime}(0)$, the slope of the kill rate function at $R=0$. For example, 


\subsection{Analysis for $\gamma=O(\epsilon)$}

It is straightforward to adapt the above analysis for the case $\gamma=O(\epsilon)$ : we seek trial solutions of the form in Equations (40) and (46) on the short and long timescales respectively. Behaviour on the short timescale $t=\epsilon \tau$ is similar to that for $\gamma=O(1)$ : binding of NK cells to tumour cells dominates the system dynamics, with changes in $A, R$ and $S$ being negligible while $C$ increases rapidly until all tumour cells are bound to NK cells (results not presented). As a result, we deduce that the initial conditions that apply on the long timescale are given by Equation (45). For $t=O(1)$ and $\gamma=\epsilon \tilde{\gamma}$, substituting with trial solutions of the form in Equation (46) in 
Equations (30)-(33) we deduce that, at leading order,

$$
\begin{aligned}
& A(t)=1+O(\epsilon), \\
& R(t)=\frac{1}{1+\alpha_{2} \tilde{\gamma}}\left(1-e^{-\theta t / \tilde{\gamma}}\right)+O(\epsilon), \\
& S(t)=1-\int_{0}^{t} \hat{f}\left(R_{0}\right) d t+O(\epsilon), \\
& C(t)=1-\int_{0}^{t} \hat{f}\left(R_{0}\right) d t+O(\epsilon),
\end{aligned}
$$

When $\gamma=\epsilon^{2} \hat{\gamma}$, we again seek trial solutions of the form given in Equations 40 and 46) on the short and long timescales respectively. In this way it is possible to derive the following leading

where $R_{K}^{*}=R_{K}\left(1+\alpha_{2} \tilde{\gamma}\right)$. Comparison of Equations (51) and (57) highlights the way in which the percentage of tumour cells killed at the end of the cytotoxicity assay $(t=1)$, particularly the nature of its dependence on the cell kill rate function $\hat{f}$, changes as $\gamma$ decreases from $\gamma=O(1)$ to $\gamma=O(\epsilon)$.

\subsection{Analysis for $\gamma=O\left(\epsilon^{2}\right)$}


order solutions for the dependent variables on the short timescale $t=\epsilon \tau$ :

$$
\begin{aligned}
& a(\tau)=1+O\left(\epsilon^{2}\right), \\
& r(\tau)=1-e^{-\tau / \hat{\gamma}}+O(\epsilon), \\
& s(\tau)=1+O(\epsilon), \\
& c(\tau)=\mu \frac{\left(1-e^{-\hat{\nu}_{1}(\mu-1) \tau}\right)}{\left(\mu-e^{-\hat{\nu}_{1}(\mu-1) \tau}\right)}+O(\epsilon),
\end{aligned}
$$

We note that the dynamics for $a, s$ and $c$ associated with the cases $\gamma=O(1)$ and $\gamma=O(\epsilon)$ are identical (see Equations (41)-44 for example). However, since initial antibody levels are now much greater than the initial number of receptors on the tumour cells, the receptors become saturated with antibody on the same short timescale as that on which the NK cells bind to the tumour cells. Matching from the short to the long timescale requires that the following initial conditions be used to determine the solutions on the long timescale:

$$
A(0)=1, R(0)=1, S(0) \text { and } C(0)=1 .
$$

Substituting with trial solutions of the form shown in Equation (46) in Equations (30)-(33) and equating to zero coefficients of $O(1)$ yields the following leading order expressions for the dependent variables:

$$
\begin{aligned}
& A(t)=1+O\left(\epsilon^{2}\right), \\
& R(t)=1-\epsilon \alpha_{2} \hat{\gamma}+O\left(\epsilon^{2}\right), \\
& S(t)=e^{-\hat{f}(1) t}+O(\epsilon), \\
& C(t)=e^{-\hat{f}(1) t}+O(\epsilon) .
\end{aligned}
$$


81

Using Equation (51) we deduce further that the percentage of tumour cells that have undergone lysis at $t=1$ is given by

$$
\left(\begin{array}{c}
\text { Percentage of tumour } \\
\text { cells killed at } t=1
\end{array}\right)=1-\frac{S(t=1)}{S(t=0)}=1-e^{-\hat{f}(1)}=1-\exp \left(\frac{-f_{K}}{1+R_{K}}\right) .
$$

Equation 66 reveals that when $\gamma=O\left(\epsilon^{2}\right)$ the percentage tumour cell kill at the end of cytotoxicity assay can be estimated by evaluating the cell kill rate function $\hat{f}(R)$ at $R=1$. Comparison of Equations 51, 57 and (66) shows further how when $\hat{f}(R)=f_{K} R /\left(R+R_{K}\right)$ the different assays provide complementary information about the parameters associated with the cell kill rate function. Combining these together gives

$$
\left(\begin{array}{c}
\text { Percentage of tumour } \\
\text { cells killed at } t=1
\end{array}\right) \approx \begin{cases}\frac{\epsilon f_{K}}{\alpha_{2} \gamma}\left(1-\frac{1}{\alpha_{2}}\left(1-e^{-\alpha_{2}}\right)\right) & \text { for } \quad \gamma=O(1), \\
f_{K}\left\{1-\frac{R_{K}^{*}}{1+R_{K}^{*}} \frac{1}{\theta} \log \left(\frac{\left(1+R_{K}^{*}\right) e^{\theta}-1}{R_{K}^{*}}\right)\right\}, & \text { for } \gamma=O(\epsilon), \\
1-\exp \left(\frac{-f_{K}}{1+R_{K}}\right), & \text { for } \gamma=O\left(\epsilon^{2}\right) .\end{cases}
$$

where $R_{K}^{*}=R_{K}\left(1+\alpha_{2} \tilde{\gamma}\right)$.

\subsection{Comparison with numerics}

In Figure 5 we compare the short and long time solutions for $R(t)$ and $C(t)$ to numerical solutions of the full model (Equations (30) - (34)). For clarity we omit the variables $A(t)$ and $S(t)$. We observe good agreement between the approximate solutions and solutions of the full model for the three cases of interest $\left(\gamma=O(1), O(\epsilon)\right.$ and $\left.O\left(\epsilon^{2}\right)\right)$.

\section{Discussion}

Antibody-based cancer therapy is now one of the most successful and important strategies for treating patients with cancer [25]. ADCC, antibody directed killing of tumour cells by natural killer cells is one of the main mechanisms of action of many clinically successful therapies [31]. 
A)

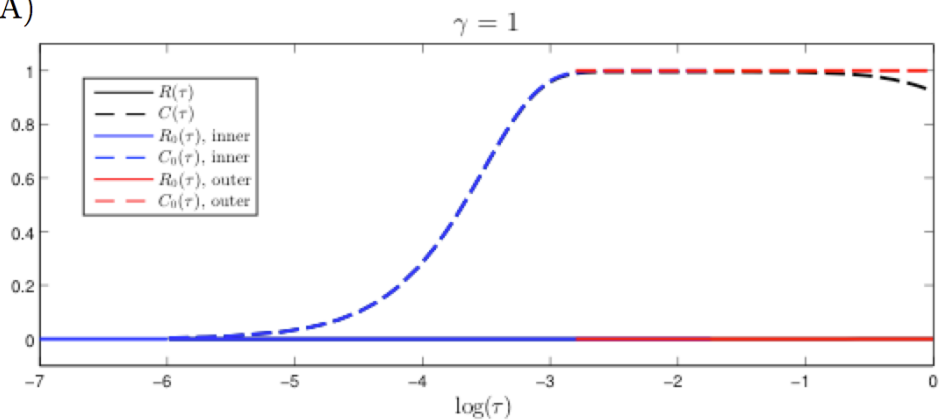

B)

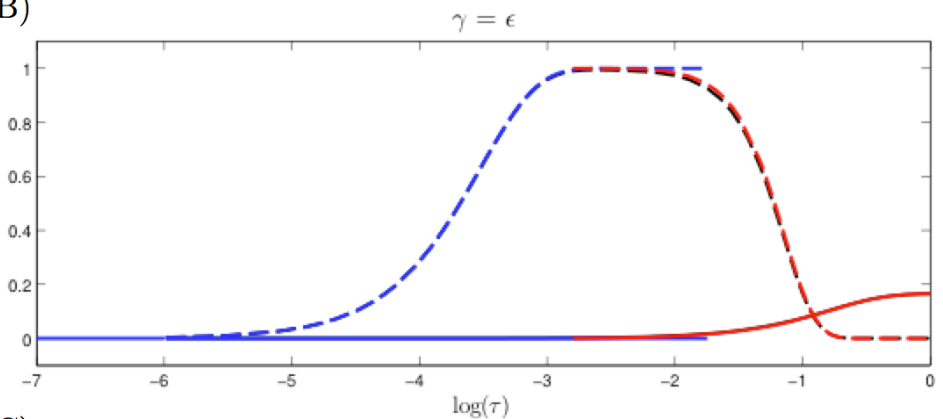

C)

$\gamma=\epsilon^{2}$

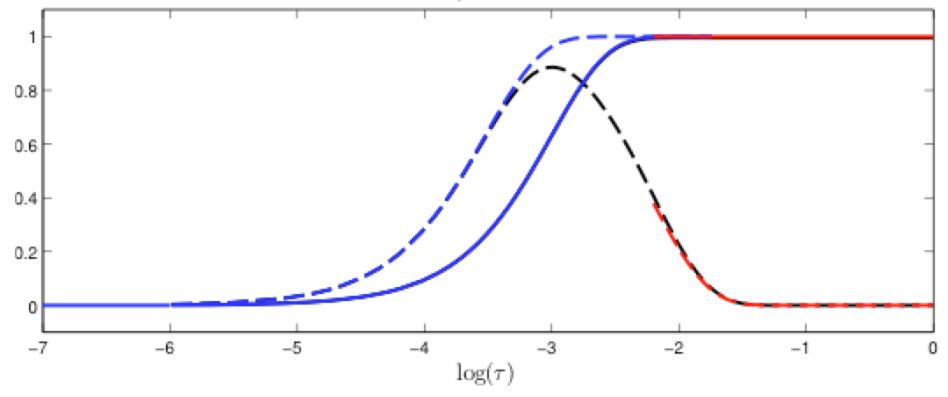

Figure 5: Series of plots showing good agreement between the approximate solutions derived in Section 4 on the short (blue curves) and long (red curves) timescales and numerical solutions of the full model (see Equations 23-26) when $\hat{f}(R)=f_{K} R /\left(R+R_{K}\right)$ and as we vary $\gamma$, the ratio of the initial number of target receptors to the initial number of antibodies. As in Figure 4 , Panel (A) corresponds to low antibody levels $(\gamma=1)$, Panel (B) to intermediate levels $(\gamma=\epsilon)$ and Panel (C) to high levels $\left(\gamma=\epsilon^{2}\right)$. In each sub-Panel, we present profiles for the number of antibody-receptor complexes per cancer cell, $R(t)=\frac{T_{b}}{T+C}$ (solid lines) and the concentration of cancer cells bound to NK cells, $C(t)$ (dashed lines). Parameter values: as per Figure 4, with (A) $\gamma=1$; (B) $\gamma=\epsilon=10^{-3}$; (C) $\gamma=\epsilon^{2}=10^{-6}$. Key: numerical solutions of the full model for $R(t)$ (black solid line) and $C(t)$ (black dashed line); leading order inner solutions for $R(t)$ (blue solid line) and $C(t)$ (blue dashed line) on the short timescale $t=O(\epsilon)$; leading solutions for $R(t)$ (red solid line) and $C(t)$ (red dashed line) on the long timescale, $t=O(1)$. 
In vitro cytotoxicity assays have been instrumental in developing ADCC mediating antibodies for clinical use.

Previous mathematical models of cytotoxicity assays, formulated as systems of time-dependent ordinary differential equations (ODEs), focussed on antibody-independent killing mechanisms and, therefore, assumed a constant kill rate [29, 30, 35, 5]. In this paper we have relaxed these assumptions to develop a new model in which tumour cell kill rates depend on how much antibody is bound to target receptors on the cancer cells. By applying perturbation methods we were able to show how information about the rate at which NK cells kill tumour cells can be extracted from in vitro cytotoxicity data.

In more detail, we used the experimental literature to estimate the model parameters and, in this way, identified two dimensionless parameters which take extremal values and a third which can vary over several orders of magnitude. In particular, the parameter $0<\alpha_{1} \ll 1$ is the product of the association rate of an antibody for its target $\left(k_{\mathrm{on}}\right)$, and the concentration of target receptors. The parameter $\nu_{1} \gg 1$ is the product of the NK-cancer cell binding rate and the initial concentration of cancer cells. Finally, the parameter $\gamma$ is the ratio of the concentration of target receptors to antibodies, and may take values across several orders of magnitude. We introduced the small parameter $0<\epsilon \ll 1$ and fixed $\alpha=\epsilon$ and $\nu_{1}=O\left(\epsilon^{-1}\right)$. We exploited the extremal values of $\alpha_{1}$ and $\nu_{1}$ to simplify the model equations, and investigated separately three cases characterised by different values of $\gamma: \gamma=O(1), O(\epsilon)$ and $O\left(\epsilon^{2}\right)$, these values of $\gamma$ reflecting the range of values of the initial ratio of antibody numbers to target receptors that are typically used in ADCC cytotoxicity assays (see Figure 3). In all cases, the system dynamics evolved on two time-scales, one fast $(t=O(\epsilon))$ and one slow $(t=O(1))$. We used perturbation methods to derive approximate expressions for the dependent variables on each time-scale and showed that these were in good agreement with numerical solutions of the full model.

The approximate solutions enabled us to relate the rate at which NK cells kill in response to 
binding antibody to the percentage of cancer cells killed at the end of a cytotoxicity assay and to determine how different initial antibody levels yield different information about the functional form of the kill rate function. This has implications for the information that can be gained about the efficacy of antibodies from cytotoxicity assays. In particular, when $\gamma=O(1)$, the concentration of antibodies is the same order of magnitude as targets, killing is negligible and proportional to $f^{\prime}(0)$, the slope of the cell kill rate function at the origin. At intermediate antibody levels, $\gamma=O(\epsilon)$, the kill rate depends on the integral of the kill rate function. Finally, when $\gamma=O\left(\epsilon^{2}\right)$, antibody levels greatly exceed target levels, targets are saturated on a fast time-scale and killing proceeds at its maximum rate, $f(1)$. Thus, in this case, information about the maximum rate of NK-cancer cell killing can be inferred.

To summarise, our asymptotic analysis reveals how measurements of lysis rates, extracted from experiments for which the ratio of initial antibody numbers to target receptors is small, could be integrated with similar data from experiments for which these ratios are intermediate and large in order to estimate the tumour cell kill rate function $\hat{f}(R)$ for a particular combination of NK cells, tumour cells and antibody. Further, numerical simulations of the full model reveal how the dynamics of the dependent variables change over time. Additional, experimental data, collected at multiple time-points, is now needed first to validate our model and then to estimate specific values of its parameters.

There is considerable scope for extending the work presented in this paper. First, we recall that in our model we assume, for simplicity, that NK cells associate and dissociate from cancer cells at constant rates, $\beta_{o n}$ and $\beta_{\text {off }}$, although Deguine et al. [8] report that antibody recognition by NK cells increases contact stability with cancer cells. It would be interesting to establish whether the qualitative behaviour of our model changes if we relax the assumption that the association and dissociation rates are constant and suppose instead that they depend on $T_{b} /(T+$ $C)$, the number of antibody-target receptor complexes per cancer cell. Additionally, we could 
consider different functional forms of the kill rate function, $f$, and a broader range of parameter regimes (see Table 3). The model could also be validated against lysis data from cytotoxicity assays performed to assess the efficacy of candidate antibodies for ADCC. More generally, the model could be applied to data for other forms of antibody dependent cell based killing such as antibody dependent cellular phagocytotosis (ADCP) involving macrophages [21]. The longterm aim of our model is to improve the ability of pharmacologists to use cytotoxicity assays to optimise antibody therapies, discriminate between experimental antibodies and design more informative experiments.

As mentioned in Sections 2 and 3, the current model is valid for the relatively short timescales associated with the cytotoxicity assay, during which processes such as cell proliferation and apoptosis can be neglected. By relaxing these assumptions, the model could be extended to describe longer time-scales on which cancer cells and NK cells proliferate as they do in vivo. The model could also be extended to capture in vivo features of tumour growth such as antibody clearance and NK cell trafficking to the cancer site, as well as the effect of other mechanisms of immune attack, angiogenesis and vascular remodelling, and other cancer treatments such as chemotherapy.

Another possible direction for further research relates to side-effects caused by the antibodies. In more detail, increasing the amount of antibodies used in the clinic has substantial draw-backs. Firstly, antibodies are typically given intravenously and are formulated at a prescribed, standard concentration, so increasing the dose means increasing the volume of antibody solution that needs to be infused. This substantially extends the time required to administer the drugs (infusion of larger volumes of drugs can take several hours) and can be extremely unpleasant to patients. Administering higher doses than strictly necessary also increases the risks of eliciting so called infusion reactions which can involve the patients immune system reacting to the foreign protein and can in extreme cases lead to anaphylaxis. For a discussion of 
infusion reactions, see Vogel et al. [33. Additionally, monoclonal antibody drugs are generally very expensive so increasing the amount administered unnecessarily increases the price of the therapy substantially.

Drug discovery is a time-consuming process which involves optimising many parameters of a pharmaceutical agent. The approach presented in this paper and the suggestions for further work illustrate some of the ways in which mathematical modelling can be used to increase the information we gain from particular experiments.

\section{Acknowledgements}

FH gratefully acknowledges funding from the ESPRC, MRC, Astrazeneca and Medimmune. We thank Professor Kevin Burrage (Queensland University of Technology), Dr Mark Penney (Medimmune, Cambridge), Professor Mark Coles (University of Leeds) and Dr Alex Phipps (Roche, UK) for helpful discussions.

\section{A Experimental Protocol}

In this appendix, we explain how the experimental data presented in Figure 1 were collected. ADCC experiments with the antibody Trastuzumab/Herceptin (Roche, Basel, Switzerland) were performed as described in [28]. In this system, Natural Killer (NK) cells were used as effector cells (E) and SkBr3 cells (a breast cancer cell line) acts as target cells (T). SkBr3 cells express high levels of the receptor protein HER2/neu on the cell surface to which Trastuzumab binds with high affinity. Briefly, peripheral blood mononuclear cells (PBMCs), a complex mixture of cells which contain NK cells, were recovered from human blood by layering over Ficoll-paque (GE Healthcare, Bucks, UK) and centrifuging at $400 \times \mathrm{g}$ for $40 \mathrm{~min}$. PBMCs were washed twice in phosphate buffered saline (PBS) and NK cells were purified by negative selection us- 
ing Robosep and the human NK cell enrichment kit (Stem Cell Technologies Inc., Vancouver, Canada) as per the manufacturer's instructions and counted with a haemocytometer.

SkBr3 cells were obtained from the American Type Culture Collection (ATCC). Cells were counted with a haemocytometer and $5 \times 10^{3}$ cells/well incubated at $37^{\circ} \mathrm{C}$ for 4 hours, together with antibody and NK effector cells at the effector to target (E:T) ratios indicated, in RPMI 1640 Glutamax I growth medium (Invitrogen, Paisley, UK) supplemented with $10 \%$ foetal bovine serum having low levels of endogenous IgG and $1 \%$ penicillin/streptomycin in a total volume of 200 l. Serving as maximum lysis control, some wells contained target cells alone and were lysed completely by the addition of $10 \mu \mathrm{l}$ of lysis agent (Cell Technology Inc., Mountain View, CA, USA) 15 min before the end of incubation and the resulting values were set as $100 \%$ lysis. Supernatant $(100 \mu \mathrm{l})$ was transferred to a 96-well Optiplate (PerkinElmer, Montreal, QC, Canada) and assayed for the presence of glyceraldehyde 3-phosphate dehydrogenase (GAPDH) levels using the aCella-Tox kit (Cell Technology Inc.) as per the manufacturer's instructions. GAPDH is an enzyme involved in glycolysis and present in all cells. It is released into the supernatants only upon lysis of cells, thus acting as a surrogate for cell killing and loss of structural integrity. All wells were corrected for background contributions from the medium by measuring wells that contained media only. Spontaneous release of GAPDH from target as well as NK cells was determined by measuring supernatants from wells containing only target cells and NK cells, respectively.

Percentage killing was calculated using the following formula in which each term refers to the amount of GAPDH released from a specific experiment:

$$
\begin{gathered}
\text { Percentage Killing }=\frac{\text { Sample }- \text { Target }_{\text {cont }}-\mathrm{NK}_{\text {cont }}-\text { Media only }}{100 \% \text { Lysis }- \text { Media only }}, \\
\text { where Target } \text { cont }=(\text { Target cells only })-(\text { Media only }),
\end{gathered}
$$


and $\mathrm{NK}_{\text {cont }}=(\mathrm{NK}$ cells only $)-($ Media only $)$.

Based on this formula, we remark that if the values for release from (target cells only) and (NK cells only) are close to those for media only then this equation can produce values that are just below zero, especially if there is some variation in the experimental values, as is almost inevitable in a biological system. This is generally not considered to be a problem as long as it is within the limits of about $5 \%$ tolerance. Many researchers would simply set these values at zero but, for reasons of scientific accuracy, we chose instead accurately to reflect the variability inherent in this assay. Finally, we note that, in Figure1, data points are mean values of triplicate measures within the representative experiment.

\section{B Parameter estimation}

In this appendix we detail how the parameters used in the model were derived.

The number of target receptors per cancer cell, $\boldsymbol{\rho}_{\boldsymbol{T}}$. In [34, Ward et al. measured the expression levels of two receptors, CD19 and CD20, which are targets of the therapeutic antibodies MEDI551 and rituximab respectively, when treating Chronic Lymphocytic Leukaemia. In a panel of patient cell lines expression levels of the two targets were in the range $O\left(10^{3}\right)-O\left(10^{4}\right)$ molecules cell ${ }^{-1}$. In [19], Kute et al. measured expression levels of HER2/neu, the target for trastuzumab when treating breast cancer. Across a panel of experimental cell lines, expression levels of HER2/neu were in the range $O\left(10^{4}\right)-O\left(10^{7}\right)$ molecules cell ${ }^{-1}$. Following [34, 19, we estimate the number of target receptors per cancer cell to be in the range $10^{3}-10^{7}$ molecules cell ${ }^{-1}$.

The antibody binding rates, $\mathbf{k}_{\mathrm{on}}$ and $\mathbf{k}_{\mathrm{off}}$. In [4] Bostrom et al. estimated the association and dissociation rates of trastuzumab, and its variants, to HER2/neu and VEGF, target 
receptors relevant for treating breast cancer. They found that $k_{\mathrm{on}}$ takes values in the range $O\left(10^{4}\right)-O\left(10^{6}\right) \mathrm{M}^{-1} \mathrm{~s}^{-1}$, while $k_{\text {off }}$ was $O\left(10^{-4}\right) \mathrm{s}^{-1}$. In [10, Ernst et al. estimated the association and dissociation rates of rituximab and its variants, for CD20, target receptors relevant for treating B cell cancers. The parameters $k_{\mathrm{on}}$ and $k_{\mathrm{off}}$ varied in the ranges $O\left(10^{3}\right)-O\left(10^{4}\right)$ $\mathrm{M}^{-1} \mathrm{~s}^{-1}$ and $O\left(10^{-4}\right)-O\left(10^{-3}\right) \mathrm{s}^{-1}$ respectively. Based on these studies, we estimate $k_{\text {on }}$ lies in the range $O\left(10^{3}\right)-O\left(10^{7}\right) \mathrm{M}^{-1} \mathrm{~s}^{-1}$ and fix $k_{\mathrm{off}}=10^{-4} \mathrm{~s}^{-1}$. We assume a fixed value for $k_{\mathrm{off}}$ for simplicity and to reduce the number of parameters that need to be considered when performing perturbation analysis (see Section 4).

NK:tumour cell association and dissociation rates, $\boldsymbol{\beta}_{\text {on }}$ and $\boldsymbol{\beta}_{\text {off }}$. In [1], Almeida et al. estimated $\beta_{\text {on }}$ and $\beta_{\text {off }}$ from in vitro conjugation assays in which approximately 200 NK cells and 200 cancer cells were co-cultured in a micro-well and the number of NK-cancer cell conjugates counted at times $t=5,10,15,20$ and 30 mins using flow cytometry. An ODE model was fit to these data to estimate the rates of conjugation and dissociation under the assumption that cell killing is negligible on this timescale. Based on this study, we fix $\beta_{\mathrm{on}}=2 \times 10^{13} \mathrm{M}^{-1} \mathrm{~s}^{-1}$ and $\beta_{\text {off }}=10^{-3} \mathrm{~s}^{-1}$

The kill-rate function, $\mathbf{f}\left(\frac{\mathbf{T}_{\mathbf{b}}}{\mathbf{T}+\mathbf{C}}\right)$. We estimate typical values of $f\left(\frac{T_{b}}{T+C}\right)$ from experimental data generated by Vanherberghen et al. [32. Using microchip-based, time-lapse imaging, they found that, once an NK:cancer cell complex forms, the time to tumour cell death was approximately $10 \mathrm{~min}$. Thus, we estimate that $f\left(\frac{T_{b}}{T+C}\right)$ takes values in the range $0-1.6 \times$ $10^{-3} \mathrm{~s}^{-1}$ 


\section{References}

[1] CR Almeida, A Ashkenazi, G Shahaf, D Kaplan, DM Davis and R Mehr (2011). Human NK cells differ more in their KIR2DL1-dependent thresholds for HLA-Cw6-mediated inhibition than in their maximal killing capacity. PLoS One 6 (9): e24927.

[2] P Bachireddy (2012). Immunology in the clinic review series; focus on cancer: multiple roles for the immune system in oncogene addiction. Clin Exp Immunol 167 (2): 188-194.

[3] R Bhat and C Watzl (2007). Serial killing of tumor cells by human natural killer cellsenhancement by therapeutic antibodies. PLoS One 2: e326.

[4] J Bostrom, L Haber, P Koenig, RF Kelley and G Fuh (2011). High affinity antigen recognition of the dual specific variants of herceptin is entropy-driven in spite of structural plasticity. PLoS One 6: e17887.

[5] DM Callewaert, DF Johnson and J Kearney (1978). Spontaneous cytotoxicity of cultured human cell lines mediated by normal peripheral blood lymphocytes. III. Kinetic parameters. J Immunol 121: 710-717.

[6] G Chu (1978). The kinetics of target cell lysis by cytotoxic T lymphocytes: A description by Poisson statistics. J Immunol 120: 1261-1267.

[7] J Deguine, B Breart, F Lematre, JP Di Santo and P Bousso (2010). Intravital Imaging Reveals Distinct Dynamics for Natural Killer and CD8+ T Cells during Tumor Regression. Immunity 33: 632-644.

[8] J Deguine, B Breart, F Lematre and P Bousso (2012). Cutting Edge: Tumor Targeting Antibodies Enhance NKG2D Mediated NK Cell Cytotoxicity by Stabilizing NK Cell Tumor Cell Interactions. J Immunol 189: 5493-5497. 
[9] EF Douglass, CJ Miller, G Sparer, H Shapiro and DA Spiegel (2013). A Comprehensive Mathematical Model for Three-Body Binding Equilibria. J Amer Chem Soc 135 (16): 60926099.

[10] JA Ernst, H Li, HS Kim, GR Nakamura, DG Yansura and RL Vandlen (2005). Isolation and characterization of the B-cell marker CD20. Biochemistry 44: 15150-15158.

[11] J Fogh, JM Fogh and T Orfeo (1977). One hundred and twenty-seven cultured human tumor cell lines producing tumors in nude mice. J Natl Cancer Inst 59 (1): 221-226.

[12] P Garcia-Penarrubia, N Lorenzo, J Galvez, A Campos, X Ferez and G Rubio (2002). Study of the physical meaning of the binding parameters involvedin effectortarget conjugation using monoclonal antibodies against adhesion molecules and cholera toxin. Cellular Immunology 215 (2): 141-150.

[13] C Gardner (2009). Stochastic Methods: A Handbook for the Natural and Social Sciences. Springer.

[14] D Hanahan and RA Weinberg RA (2011). Hallmarks of Cancer: The Next Generation. Cell 144 (5): 646-674.

[15] FS Hodi FS, SJ O’Day, DF McDermott, RW Weber, JA Sosman, JB Haanen, R Gonzalez, C Robert, D Schadendorf, JC Hassel, W Akerley, AJ van den Eertwegh, J Lutzky, P Lorigan, JM Vaubel, GP Linette, D Hogg, CH Ottensmeier, C Lebb, C Peschel, I Quirt, JI Clark, JD Wolchok, JS Weber, J Tian, MJ Yellin, GM Nichol, A Hoos and WJ Urba (2010). Improved survival with ipilimumab in patients with metastatic melanoma. $N$ Engl J Med 363: 711.

[16] CA Hudis (2007). Trastuzumab-mechanism of action and use in clinical practice. $N$ Engl $J$ Med 357 (1): 39-51. 
[17] S Iida, R Kuni-Kamochi, K Mori, H Misaka, M Inoue, A Okazaki, K Shitara and M Satoh (2009). Two mechanisms of the enhanced antibody-dependent cellular cytotoxicity (ADCC) efficacy of non-fucosylated therapeutic antibodies in human blood. BMC Cancer 9: 58.

[18] CT Lutz, A Karapetyan, A Al-Attar, BJ Shelton, KJ Holt, JH Tucker and SR Presnell (2011). Human NK Cells Proliferate and Die In Vivo More Rapidly than T Cells in Healthy Young and Elderly Adults. J Immunol 186 (8): 4590-4598.

[19] T Kute, JR Stehle Jr, D Ornelles, N Walker, O Delbono and JP Vaughn (2012). Understanding key assay parameters that affect measurements of trastuzumab-mediated ADCC against Her2 positive breast cancer cells. OncoImmunology 1 (6): 810-821.

[20] Mathworks (2013). Matlab 2013a. http://www.mathworks.co.uk

[21] K Matlawska-Wasowska, E Ward, S Stevens, Y Wang, R Herbst, SS Winter and BS Wilson (2013). Macrophage and NK-mediated killing of precursor-B acute lymphoblastic leukemia cells targeted with a-fucosylated anti-CD19 humanized antibodies. Leukemia 27 (6): 12631274 .

[22] RG Miller and M Dunkley (1974). Quantitative analysis of the 51Cr release cytotoxicity assay for cytotoxic lymphocytes Cell Immunol 14: 284-302.

[23] S Nakagawa, T Fujii, G Yokoyama, MG Kazanietz, H Yamana and K Shirouzu (2003). Cell growth inhibition by all-trans retinoic acid in SKBR-3 breast cancer cells: Involvement of protein kinase $\mathrm{Ca}$ and extracellular signal-regulated kinase mitogen-activated protein kinase Mol Carcinogenesis 38 (3): 106-116.

[24] H Ogbomo, A Hahn, J Geiler, M Michaelis, HW Doerr and J Cinatl Jr (2006). NK sensitivity of neuroblastoma cells determined by a highly sensitive coupled luminescent method. Biochem Biophys Res Comm 339 (1): 375-379. 
[25] AM Scott, JD Wolchok and LJ Old (2012). Antibody therapy of cancer. Nat Rev Cancer 12: $278-287$.

[26] UJE Seidel, P Schlegel and P Lang (2013). Natural killer cell mediated antibody-dependent cellular cytotoxicity in tumor immunotherapy with therapeutic antibodies. Front Immunol 4: 76 .

[27] LF Shampine and MW Reichelt MW (1997). The MATLAB ODE Suite. SIAM J Sci Comput 18: 1-22.

[28] R Stewart, G Thom, M Levens, G Guler-Gane, R Holgate, PM Rudd, C Webster, L Jermutus and J Lund (2011). A variant human IgG1-Fc mediates improved ADCC Protein Eng Des Sel 24 (9): 671-678.

[29] RM Thorn and CS Henney (1976). Kinetic analysis of target cell destruction by effector T cells. I. Delineation of parameters related to the frequency and lytic efficiency of killer cells. J Immunol 117: 2213-2219.

[30] RM Thorn and CS Henney (1977). Kinetic analysis of target cell destruction by effector T cells. II. Changes in killer cell avidity as a function of time and antigen dose. J Immunol 119: 1973-1978.

[31] E Vacchelli, J Pol, N Bloy, A Eggermont, I Cremer, WH Fridman, J Galon, A Marabelle, H Kohrt, L Zitvogel, G Kroemer and L Galluzzi (2015). Trial watch: tumour-targetting monoclonal antibodies for oncological indications. Oncoimmunology 4: e985940.

[32] B Vanherberghen, PE Olofsson, E Forslund, M Sternberg-Simon, MA Khorshidi, S Pacouret, K Guldevall, M Enqvist, KJ Malmberg, R Mehr and B nfelt (2013). Classification of human natural killer cells based on migration behavior and cytotoxic response. Blood 121 (8): 1326-1334. 
[33] WH Vogel (2010). Infusion reactions: diagnosis, assessment, and management. Clin J Oncol Nurs 14: E10-21.

[34] E Ward, N Mittereder, E Kuta, GP Sims, MA Bowen, W Dall'Acqua, T Tedder, P Kiener, AJ Coyle, H Wu, B Jallal and R Herbst (2011). A glycoengineered anti-CD19 antibody with potent antibody-dependent cellular cytotoxicity activity in vitro and lymphoma growth inhibition in vivo. Br J Haem 155 (4): 426-437.

[35] WP Zeijlemaker, RHJ Van Oers, REY De Goede and PTA Schellekens (1977). Cytotoxic activity of human lymphocytes: quantitative analysis of $\mathrm{T}$ cell and $\mathrm{K}$ cell cytotoxicity, revealing enzyme-like kinetics. J Immunol 119: 1507-1514. 